Research Article

\title{
Qualitative Analysis of the Effect of Weeds Removal in Paddy Ecosystems in Fallow Season
}

\author{
Leru Zhou ${ }^{1}{ }^{1}{ }^{1}$ Zhigang Liu $\mathbb{D}^{2}{ }^{2}$ and Tiejun Zhou $\mathbb{D}^{2}$ \\ ${ }^{1}$ College of Resources and Environment, Fujian Agriculture and Forestry University, Fuzhou, Fujian 350002, China \\ ${ }^{2}$ College of Information and Intelligence Science, Hunan Agricultural University, Changsha, Hunan 410128, China
}

Correspondence should be addressed to Tiejun Zhou; hntjzhou@126.com

Received 26 June 2020; Revised 7 October 2020; Accepted 23 October 2020; Published 11 November 2020

Academic Editor: Leonid Shaikhet

Copyright (c) 2020 Leru Zhou et al. This is an open access article distributed under the Creative Commons Attribution License, which permits unrestricted use, distribution, and reproduction in any medium, provided the original work is properly cited.

In the paper, we introduce a differential equations model of paddy ecosystems in the fallow season to study the effect of weeds removal from the paddy fields. We found that there is an unstable equilibrium of the extinction of weeds and herbivores in the system. When the intensity of weeds removal meets certain conditions and the intrinsic growth rate of herbivores is higher than their excretion rate, there is a coexistence equilibrium state in the system. By linearizing the system and using the Routh-Hurwitz criterion, we obtained the local asymptotically stable conditions of the coexistence equilibrium state. The critical value formula of the Hopf bifurcation is presented too. The model demonstrates that weeds removal from paddy fields could largely reduce the weeds biomass in the equilibrium state, but it also decreases the herbivore biomass, which probably reduces the content of inorganic fertilizer in the soil. We found a particular intensity of weeds removal that could result in the minimum content of inorganic fertilizer, suggesting weeds removal should be kept away from this intensity.

\section{Introduction}

Paddy field fertility is the premise of high quality, high yield, and low energy consumption of rice. The method of increasing rice yield by applying a large amount of chemical fertilizers and pesticides has threatened the sustainable development of grain and the ecological security. Therefore, how to use biodiversity in paddy ecosystems to improve the stability and sustainability of agricultural systems has been attracting more and more attention [1-3].

There are many factors affecting the fertility of a paddy ecosystem, such as soil nutrient availability, light, moisture, weeds, insects, microorganisms, and so on. Not only the number of these factors is big but also the relations among them are complicated. In addition, there are many human disturbances in a paddy ecosystem. Because of these problems, the current research focuses on the experiments and data analyses of paddy ecosystems but rarely on the dynamics of the systems by establishing mathematical models. For example, through experiments, it is found that putting ducks in rice fields could improve soil fertility, control weeds, and reduce rice diseases [4-7]. Our interest is in using models of differential equations to study the complex nonlinear relationships in paddy ecosystems. For the forest and aquatic ecosystems, some matured mathematical models have been established [8-26]. For example, Hofmann and Ambler developed a system consisting of ten coupled ordinary differential equations to describe the interactions among nitrate, ammonium, two phytoplankton size components, five copepods, and a debris pool on the continental shelf outside the southeastern United States [13]. For an aquatic ecosystem, it is very important to explore the reproductive mechanism of phytoplankton. Because periodic solutions in mathematics can represent reproduction, many scholars have studied the existences of periodic solutions, almost periodic solutions, and Hopf bifurcations of some aquatic ecosystems $[8,10,12,14,17-19,27]$, and some scholars have studied the effect of time delay on nutrient metabolism kinetics [17].

In recent years, we have established some mathematical models of paddy ecosystems from different aspects [28-32]. We built a differential equations model of a paddy ecosystem 
in fallow season in [28], and studied the interaction between weeds and soil nutrient availability with the discovery of the existence of the stable node, unstable saddle point, or saddlenode in the system. Xiang et al. found that adding the factor of domesticated animals in a paddy ecosystem in the fallow season could improve the level of nutrient availability in the soil [29], and some new features such as a Hopf bifurcation appear in the system.

In addition to farming animals in paddy fields, weeds removal is also a common activity of farmland management. Farmers gather weeds from paddy fields to reduce the consumption of soil nutrients and to feed animals too. Removing weeds from the paddy fields has some unavoidable impacts on the paddy ecosystem. We are interested in how the factors in a paddy ecosystem are affected by this activity and what principles should be followed to measure it. The main purpose of this paper is to analyze the effect of weeds removal in a paddy ecosystem in the fallow season by establishing a differential equations model and find a strategy of weeds removal.

The rest of the paper is organized as follows. In the next section, the mathematical model of the paddy ecosystem with weeds removal is introduced. We present the conditions of existence of equilibria in Section 3. Then, we consider the stability of equilibria in Section 4. In Section 5, we analyze the effect of weeds removal on the main factors in the paddy ecosystem. We study the Hopf bifurcation from the coexistence equilibrium in Section 6. Some numerical simulations are carried out in Section 7. Finally, we give our conclusion.

\section{Modeling of the Paddy Ecosystem in the Fallow Season with Weeds Removal}

We only consider the effect of weeds removal on three main components of a paddy ecosystem in fallow season: weeds, inorganic fertilizer, and herbivores. There is an obvious nutrient-dependent consumption ring among the three: weeds are used as food for farmed animals in paddy fields, weeds are nourished by the effective nutrients in the soil, and the effective nutrients can be transformed from animal manure. In [29], Xiang et al. proposed the following differential equation model of a paddy ecosystem in the fallow season to analyze the interactions among the three main components:

$$
\left\{\begin{array}{l}
\dot{p}(t)=f(I) H(u) p-h(p) A-d_{1} p, \\
\dot{u}(t)=-f(I) H(u) p+(1-r) h(p) A+d_{1} p+d_{2} A, \\
\dot{A}(t)=b A\left(1-\frac{A}{K}\right)+r h(p) A-d_{2} A,
\end{array}\right.
$$

where $p(t)$ is the weeds biomass per unit area at time $t, u(t)$ is the inorganic fertilizer content per unit area, and $A(t)$ is the herbivore biomass per unit area. The function $f(I)=$ $\left(f_{m} I /\left(I_{k}+I\right)\right)$ is a light effect function. The function
$H(u)=(a u /(m+u))$ is used as Michaelis-Menten uptake kinetics of the general form, where $m$ is the half saturation concentration of inorganic fertilizer and $a$ is the maximum uptake rate of inorganic fertilizer $u$. The function

$$
h(p)=c\left(1-e^{-\lambda p}\right)
$$

is the rate of grazing weeds per herbivore, where $c$ is the maximum rate of grazing weeds by a herbivore and $\lambda$ is a constant affecting the grazing rate. The three terms on the right of the first equation in system (1), respectively, represent the growth of weeds, the herbivore grazing, and the natural mortality of weeds, which the first term $f(I) H(u) p$ shows the weeds growth is affected by the light intensity $I$ and the inorganic fertilizer $u, d_{1}$ is the mortality rate of weeds. There are four terms on the right hand side of the second equation in system (1), the first term is the consumption of inorganic fertilizer by the growth of weeds, the second term represents the inorganic fertilizer converted from some weeds that have been wasted by the herbivore, the third term is the inorganic fertilizers transformed from dead weeds, and the last term is herbivore excrements. The three terms on the right hand side of the last equation in system (1) represent in turn the growth of herbivore, the herbivore assimilating of weeds and herbivore excretion, where $b$ is the intrinsic growth rate of herbivore and $K$ is the largest environmental capacity of herbivore, $r$ is the assimilating ratio of herbivore, and $d_{2}$ is the excretion rate of herbivore.

To consider the effect of weeds removal from the paddy fields, we introduce the weeds removal term $d_{0} p$ into the first equation of the model (1),

$$
\left\{\begin{array}{l}
\dot{p}(t)=f(I) H(u) p-h(p) A-d_{0} p-d_{1} p \\
\dot{u}(t)=-f(I) H(u) p+(1-r) h(p) A+d_{1} p+d_{2} A \\
\dot{A}(t)=b A\left(1-\frac{A}{K}\right)+r h(p) A-d_{2} A .
\end{array}\right.
$$

The third term $d_{0} p$ on the right of the first equation represents the rate of artificial weeds removal and $d_{0}$ is called the weeds removal intensity.

To reduce the system parameters, we make the following transformations, $\quad \bar{p}=(p / K), \quad \bar{u}=(u / K), \bar{A}=(A / K)$, $\bar{m}=(m / K), \bar{a}=f(I) a$, and $\bar{\lambda}=\lambda K$. System (3) thereby is in the following form:

$$
\left\{\begin{array}{l}
\dot{p}(t)=H(u) p-h(p) A-d_{0} p-d_{1} p, \\
\dot{u}(t)=-H(u) p+(1-r) h(p) A+d_{1} p+d_{2} A \\
\dot{A}(t)=b A(1-A)+r h(p) A-d_{2} A .
\end{array}\right.
$$

In system (4), we have removed the bar above some variables or parameters. Note that the parameter $a$ in the function $H(u)$ represents both the light effect and the uptake rate of inorganic fertilizer. Here, we call the parameter $a$ the coupling effect coefficient of light and fertilizer.

In the sense of ecology, the parameters in system (4) are nonnegative, and $0<r<1, d_{1}>0$, and $d_{2}>0$. The properties 
of paddy ecosystem without weeds removal have been studied in [29], so we only consider the case $d_{0}>0$.

\section{The Existence of Equilibria}

Denote the three expressions on the right hand side of system (4) by

$$
\begin{aligned}
& F_{1}(p, u, A)=H(u) p-h(p) A-d_{0} p-d_{1} p, \\
& F_{2}(p, u, A)=-H(u) p+(1-r) h(p) A+d_{1} p+d_{2} A, \\
& F_{3}(p, u, A)=b A(1-A)+r h(p) A-d_{2} A .
\end{aligned}
$$

In order to obtain the equilibrium states, we need to solve the nonlinear equations $F_{i}(p, u, A)=0(i=1,2,3)$. Accordingly, we have $F_{1}(p, u, A)+F_{2}(p, u, A)+F_{3}(p, u, A)=0$, or

$$
d_{0} p=b A(1-A) \text {. }
$$

From (6), we know $0 \leq A \leq 1$.

According to $F_{3}(p, u, A)=A\left(b(1-A)+r h(p)-d_{2}\right)=0$, we have $A=0$ or $b(1-A)=d_{2}-r h(p)$.

If $A=0$, then $F_{1}(p, u, A)=\left(H(u)-d_{0}-d_{1}\right) p=0$ and $F_{2}(p, u, A)=\left(-H(u)+d_{1}\right) p=0$; thereby, we obtain that $p=0$. Hence, there exists one equilibrium of system (4) as follows:

$$
\left(p_{1}, u_{1}, A_{1}\right)=\left(0, u_{1}, 0\right),
$$

where $u_{1}$ is an arbitrary nonnegative real constant.

If equation $b(1-A)=d_{2}-r h(p)$ holds, we have the following:

$$
h(p)=\frac{d_{2}-b+b A}{r} .
$$

Define

$$
d(A)=\frac{1}{r}\left(d_{2}-b+b A\right)
$$

where $A \in[0,1]$. Obviously, $d(A)=h(p)$. From $h(p) \geq 0$, we know that $d(A) \geq 0$ when $A \in[0,1]$, so we have $A \geq 1-\left(d_{2} / b\right)=A_{*}$. Synthesizing (2) and (8), we obtain the following:

$$
e^{-\lambda p}=1-\frac{d(A)}{c}>0 .
$$

Thus, we have $d(A)<c$. It follows that

$$
A<1-\frac{d_{2}}{b}+\frac{r c}{b} \text {. }
$$

Denote $A^{*}=\min \left\{1,1-\left(d_{2} / b\right)+(r c / b)\right\}$. Notice that $0 \leq A \leq 1$, we have the following:

$$
\max \left\{0, A_{*}\right\} \leq A<A^{*} .
$$

Therefore, if the variable $A$ locates in the above range, then we get

$$
p=-\frac{1}{\lambda} \ln \left(1-\frac{d(A)}{c}\right) .
$$

Substituting $p$ into (6) yields

$$
d_{0} \ln \left(1-\frac{d(A)}{c}\right)=-\lambda b A(1-A) .
$$

Formula (14) is regarded as an equation of an unknown quantity $A$. For the existence of the roots of equation (14), we give the following Lemma 1.

Lemma 1. If $b>d_{2}$, then, equation (14) has at least one positive root $A_{2} \in\left(A_{*}, A^{*}\right)$.

Proof. From $b>d_{2}$ we know $A_{*}>0$. Define

$$
\varphi(A)=\lambda b A(1-A)+d_{0} \ln \left(1-\frac{d(A)}{c}\right) .
$$

It is continuous in the intervals $\left[A_{*}, A^{*}\right)$. Obviously, the value of the function $\varphi(A)$ at $A=A_{*}$ is

$$
\varphi\left(A_{*}\right)=\lambda d_{2}\left(1-\frac{d_{2}}{b}\right) .
$$

If $r c>d_{2}$, then $A^{*}=1$, and we have the following:

$$
\varphi\left(A^{*}\right)=d_{0} \ln \left(1-\frac{d_{2}}{r c}\right)<0 .
$$

If $r c \leq d_{2}$, then $A^{*}=1-\left(d_{2} / b\right)+(r c / b)$, and we have the following:

$$
\begin{aligned}
\lim _{A \longrightarrow A^{*}-0} \varphi(A)= & \lambda\left(1-\frac{d_{2}}{b}+\frac{r c}{b}\right)\left(d_{2}-r c\right) \\
& +d_{0} \lim _{A \longrightarrow A^{*}-0} \ln \left(1-\frac{d_{2}-b+b A}{r c}\right)=-\infty .
\end{aligned}
$$

Thus, the function $\varphi(A)$ must have a zero point $A_{2} \in\left(A_{*}, A^{*}\right)$. Therefore, equation (14) has at least one positive root $A_{2} \in\left(A_{*}, A^{*}\right)$.

We make the following hypothesis on the positive root $A_{2}$,

(H1). $a>d_{0}+d_{1}+d_{0} d\left(A_{2}\right) /\left(b\left(1-A_{2}\right)\right)$.

Substituting $A=A_{2}$ into (6) yields

$$
p_{2}=\frac{b}{d_{0}} A_{2}\left(1-A_{2}\right) \text {. }
$$

By substituting $A=A_{2}$ and $p=p_{2}$ into $F_{1}(p, u, A)=0$, we get

$$
\begin{aligned}
H(u) & =\frac{a u}{m+u}=d_{0}+d_{1}+\frac{A_{2} h\left(p_{2}\right)}{p_{2}} \\
& =d_{0}+d_{1}+\frac{A_{2} d\left(A_{2}\right)}{p_{2}} .
\end{aligned}
$$

Then, we obtain

$$
u=\frac{m\left(b\left(1-A_{2}\right)\left(d_{0}+d_{1}\right)+d_{0} d\left(A_{2}\right)\right)}{b\left(1-A_{2}\right)\left(a-d_{0}-d_{1}\right)-d_{0} d\left(A_{2}\right)} \triangleq u_{2} .
$$


Notice that $d(A)>0$ for $A \in\left[A_{*}, A^{*}\right)$, it follows that the numerator of $u_{2}$ is positive. Denote the denominator of $u_{2}$ by

$$
g\left(A_{2}\right)=b\left(1-A_{2}\right)\left(a-d_{0}-d_{1}\right)-d_{0} d\left(A_{2}\right) .
$$

Obviously, if the assumption (H1) holds, then the denominator

$$
g\left(A_{2}\right)=b\left(1-A_{2}\right)\left(a-d_{0}-d_{1}-\frac{d_{0} d\left(A_{2}\right)}{b\left(1-A_{2}\right)}\right)>0 .
$$

Therefore, we deduce that $u_{2}>0$.

Based on the above analysis, we obtain the following conclusions about the existence of equilibria in system (4).

Theorem 1. System (4) has weeds and herbivore extinction equilibrium $\left(p_{1}, u_{1}, A_{1}\right)=\left(0, u_{1}, 0\right)$. If conditions $b>d_{2}$, and (H1) hold, there also exists at least one positive equilibrium $E_{p}=\left(p_{2}, u_{2}, A_{2}\right)$, where $u_{1}$ is an arbitrary nonegative real constant, $A_{2}$ is one positive root of equation (14), and $p_{2}$ and $u_{2}$ are given in (19) and (21), respectively.

The biological significance of condition $b>d_{2}$ in Lemma 1 and Theorem 1 is that the intrinsic growth rate $b$ of herbivore should be greater than its excretion rate $d_{2}$. The use of hypothesis $(\mathrm{H} 1)$ is to ensure that $u_{2}$ is positive. Regarding hypothesis $(\mathrm{H} 1)$, let us make a further explanation. If we set

$$
A^{* *}=1-\frac{d_{0} d_{2}}{b\left(r\left(a-d_{0}-d_{1}\right)+d_{0}\right)},
$$

then hypothesis (H1) is equivalent to

$$
\begin{aligned}
a & >d_{0}+d_{1}, \\
A_{2} & <A^{* *} .
\end{aligned}
$$

Obvousily, $A^{* *}>A_{*}$. But the relationship between $A^{* *}$ and $A^{*}$ is complex. If $d_{2} \leq r c$, then $A^{*}=1>A^{* *}$. If $d_{2}>r c$, then $A^{*}=1-\left(d_{2} / b\right)+(r c / b)$. Furthermore, if $d_{0}>\left(a-d_{1}\right) /\left(1+c /\left(d_{2}-r c\right)\right)$, then we have $A^{*}>A^{* *}$. Otherwise, $A^{*} \leq A^{* *}$. Therefore, if conditions

$$
\begin{aligned}
& d_{2}>r c, \\
& d_{0} \leq \frac{a-d_{1}}{1+c /\left(d_{2}-r c\right)},
\end{aligned}
$$

hold, then we may not need a hypothesis (H1). That is to say, (H1) should be assumed only if $d_{2} \leq r c$ or $d_{2}>r c$ and $d_{0}>\left(a-d_{1}\right) /\left(1+c /\left(d_{2}-r c\right)\right)$.

Generally speaking, in order to ensure the normal growth of herbivore, its excretion rate $d_{2}$ should not be greater than its assimilation rate $r c$ of weeds (that is $d_{2} \leq r c$ ). The case of " $d_{2}>r c$ " may appear in the period when the herbivore is sick. Therefore, under normal circumstances, condition (H1) is necessary to ensure the existence of positive equilibrium.

\section{The Stability of Equilibria}

Now, let us discuss the stabilities of equilibrium $\left(p^{*}, u^{*}, A^{*}\right)$ of system (4). With a coordinate transformation $x=p-p^{*}$, $y=u-u^{*}, z=A-A^{*}$, system (4) is converted to

$$
\left\{\begin{array}{l}
\dot{x}(t)=-\left(d_{0}+d_{1}\right) x(t)+\left[H\left(y+u^{*}\right)\left(x+p^{*}\right)-H\left(u^{*}\right) p^{*}\right]-\left[h\left(x+p^{*}\right)\left(z+A^{*}\right)-h\left(p^{*}\right) A^{*}\right] \\
\dot{y}(t)=d_{1} x(t)+d_{2} z(t)-\left[H\left(y+u^{*}\right)\left(x+p^{*}\right)-H\left(u^{*}\right) p^{*}\right]+(1-r)\left[h\left(x+p^{*}\right)\left(z+A^{*}\right)-h\left(p^{*}\right) A^{*}\right] \\
\dot{z}(t)=\left(b-2 b A^{*}-d_{2}\right) z-b z^{2}+r\left[h\left(x+p^{*}\right)\left(z+A^{*}\right)-h\left(p^{*}\right) A^{*}\right]
\end{array}\right.
$$

The linearized system of (27) at the equilibrium $(0,0,0)$ is as follows:

$$
\left\{\begin{array}{l}
\dot{x}(t)=\left[H\left(u^{*}\right)-h^{\prime}\left(p^{*}\right) A^{*}-d_{0}-d_{1}\right] x+p^{*} H^{\prime}\left(u^{*}\right) y-h\left(p^{*}\right) z \\
\dot{y}(t)=\left[d_{1}-H\left(u^{*}\right)+(1-r) h^{\prime}\left(p^{*}\right) A^{*}\right] x-p^{*} H^{\prime}\left(u^{*}\right) y+\left[d_{2}+(1-r) h\left(p^{*}\right)\right] z \\
\dot{z}(t)=r h^{\prime}\left(p^{*}\right) A^{*} x+\left(b-2 b A^{*}+r h\left(p^{*}\right)-d_{2}\right) z
\end{array}\right.
$$

where $H^{\prime}(u)=\left(a m /(m+u)^{2}\right)$, and $h^{\prime}(p)=c \lambda e^{-\lambda p}$.

The stability of the equilibrium $\left(p_{1}, u_{1}, A_{1}\right)=\left(0, u_{1}, 0\right)$ of system (4) is presented in Theorem 2.

Theorem 2. The equilibrium $\left(p_{1}, u_{1}, A_{1}\right)=\left(0, u_{1}, 0\right)$ of system (4) is unstable.
Proof. For the equilibrium $\left(p_{1}, u_{1}, A_{1}\right)=\left(0, u_{1}, 0\right)$, we have $h\left(p_{1}\right)=h(0)=0$ and $h^{\prime}\left(p_{1}\right)=h^{\prime}(0)=c \lambda$, so the linearized system (28) becomes as follows:

$$
\left\{\begin{array}{l}
\dot{x}(t)=\left[H\left(u^{*}\right)-d_{0}-d_{1}\right] x \\
\dot{y}(t)=\left[d_{1}-H\left(u^{*}\right)\right] x+d_{2} z \\
\dot{z}(t)=\left(b-d_{2}\right) z
\end{array}\right.
$$


Its solution under initial condition $(x(0), y(0), z(0))$ is as follows:

$$
\begin{aligned}
x(t)= & x(0) e^{\left(H\left(u^{*}\right)-d_{0}-d_{1}\right) t}, \\
z(t)= & z(0) e^{\left(b-d_{2}\right) t}, \\
y(t)= & \frac{x(0)\left(d_{1}-H\left(u^{*}\right)\right)}{H\left(u^{*}\right)-d_{0}-d_{1}} e^{\left(H\left(u^{*}\right)-d_{0}-d_{1}\right) t} \\
& +\frac{z(0) d_{2}}{b-d_{2}} e^{\left(b-d_{2}\right) t}+y(0) .
\end{aligned}
$$

It shows that the equilibrium $(0,0,0)$ of system $(29)$ is unstable. Therefore, the equilibrium $\left(p_{1}, u_{1}, A_{1}\right)=\left(0, u_{1}, 0\right)$ of system (4) is also unstable.

Next, we discuss the stability of the positive equilibrium $E_{p}$. For convenience, we introduce the following symbols to express the coefficients of system (28),

$$
\begin{aligned}
& a_{11}=H\left(u_{2}\right)-A_{2} h^{\prime}\left(p_{2}\right)-d_{0}-d_{1}, \\
& a_{12}=p_{2} H^{\prime}\left(u_{2}\right), \\
& a_{21}=-H\left(u_{2}\right)+(1-r) A_{2} h^{\prime}\left(p_{2}\right)+d_{1}, \\
& a_{23}=d_{2}+(1-r) h\left(p_{2}\right), \\
& a_{31}=r A_{2} h^{\prime}\left(p_{2}\right), \\
& a_{33}=b-2 b A_{2}+r h\left(p_{2}\right)-d_{2}, \\
& a_{13}=-h\left(p_{2}\right) \\
& a_{22}=-a_{12} .
\end{aligned}
$$

Obviously, $a_{21}=-a_{11}-a_{31}-d_{0}$. At the positive equilibrium $E_{p}$, we have $b\left(1-A_{2}\right)=d_{2}-r h\left(p_{2}\right)$ and $h\left(p_{2}\right)=d\left(A_{2}\right)$. Thus, we obtain the following:

$$
\begin{aligned}
& a_{13}=-d\left(A_{2}\right), \\
& a_{23}=d\left(A_{2}\right)+b\left(1-A_{2}\right), \\
& a_{33}=-b A_{2} .
\end{aligned}
$$

Considering the following facts:

$$
\begin{aligned}
& H^{\prime}\left(u_{2}\right)=\frac{a m}{\left(m+u_{2}\right)^{2}}>0, \\
& h^{\prime}\left(p_{2}\right)=c \lambda e^{-\lambda p_{2}}=\lambda\left(c-d\left(A_{2}\right)\right)>0,
\end{aligned}
$$

it is easy to see that $a_{12}$ and $a_{31}$ are all positive. As for the positive and negative properties of $a_{11}$ and $a_{21}$, we have the following conclusions.

Lemma 2. At the coexistence equilibrium $E_{p}, a_{11}>0$ and $a_{21}<0$.

Proof. Let

$$
D_{0}\left(A_{2}\right) \triangleq d\left(A_{2}\right)-\lambda p_{2}\left(c-d\left(A_{2}\right)\right) .
$$

From in (13), we have

$$
\begin{aligned}
D_{0}\left(A_{2}\right) & =d\left(A_{2}\right)+\left(c-d\left(A_{2}\right)\right) \ln \left(1-\frac{d\left(A_{2}\right)}{c}\right) \\
& =\left(c-d\left(A_{2}\right)\right)\left[\frac{d\left(A_{2}\right)}{c-d\left(A_{2}\right)}+\ln \left(1-\frac{d\left(A_{2}\right)}{c}\right)\right] .
\end{aligned}
$$

Notice that $c>d\left(A_{2}\right)$, we get the following result by Lemma 2.1 in [29].

$$
-\ln \left(1-\frac{d\left(A_{2}\right)}{c}\right)<\frac{d\left(A_{2}\right) / c}{1-d\left(A_{2}\right) / c}=\frac{d\left(A_{2}\right)}{c-d\left(A_{2}\right)} .
$$

It follows that $D_{0}\left(A_{2}\right)>0$.

From $F_{1}\left(p_{2}, u_{2}, A_{2}\right)=H\left(u_{2}\right) p_{2}-A_{2} h\left(p_{2}\right)-\left(d_{0}+d_{1}\right)$ $p_{2}=0, h\left(p_{2}\right)=d\left(A_{2}\right)$, we have

$$
H\left(u_{2}\right)-d_{0}-d_{1}=\frac{A_{2} d\left(A_{2}\right)}{p_{2}} .
$$

Therefore,

$a_{11}=\frac{A_{2} d\left(A_{2}\right)}{p_{2}}-A_{2} h^{\prime}\left(p_{2}\right)=\frac{A_{2}}{p_{2}}\left(d\left(A_{2}\right)-p_{2} h^{\prime}\left(p_{2}\right)\right)$.

Substituting (33) into the expression of $a_{11}$ yields

$$
a_{11}=\frac{A_{2} D_{0}\left(A_{2}\right)}{p_{2}} \text {. }
$$

Therefore, $a_{11}>0$.

From (31) and (33), we obtain that

$$
\begin{aligned}
a_{21} & =-\left(a_{11}+d_{0}\right)-a_{31} \\
& =-\left(a_{11}+d_{0}\right)-r A_{2} h^{\prime}\left(p_{2}\right) \\
& =-\left(a_{11}+d_{0}\right)-r \lambda\left(c-d\left(A_{2}\right)\right)<0 .
\end{aligned}
$$

We introduce the following notations:

$$
\left\{\begin{array}{l}
a_{1}=a_{12}-a_{11}+b A_{2}, \\
a_{2}=b A_{2}\left(a_{12}-a_{11}\right)+a_{12} d_{0}+a_{31}\left(d\left(A_{2}\right)+a_{12}\right), \\
a_{3}=a_{12} d_{0} b A_{2}+a_{12} a_{31} b\left(2 A_{2}-1\right),
\end{array}\right.
$$

and define a quadratic function

$$
J(A)=-2 b \lambda A^{2}+\lambda\left(2 r c-2 d_{2}+3 b\right) A+d_{0}-\lambda\left(r c-d_{2}+b\right) \text {. }
$$

It is easy to verify that $\lim _{A \longrightarrow \pm \infty} J(A)=-\infty$.

If $r c>d_{2}$, then

$$
J\left(A^{*}\right)=J(1)=\lambda\left(r c-d_{2}\right)+d_{0}>0,
$$

otherwise

$$
J\left(A^{*}\right)=J\left(1-\frac{d_{2}}{b}+\frac{r c}{b}\right)=d_{0}>0 .
$$

Therefore, the quadratic equation $J(A)=0$ has two real roots $A^{-}<A^{*}$ and $A^{+}>A^{*}$. 
According to the properties of polynomial $J(A)$, we can determine whether $a_{3}$ defined in (41) is a positive number.

Lemma 3. If $A_{2}>A^{-}$, then $a_{3}>0$. Otherwise, if $A_{2}<A^{-}$, then $a_{3}<0$.

Proof. According to the definition of $a_{31}$ in (31), by replacing $h^{\prime}\left(p_{2}\right)$ with (33), we get the following:

$$
\begin{aligned}
a_{3}= & a_{12} b\left[d_{0} A_{2}+r A_{2} h^{\prime}\left(p_{2}\right)\left(2 A_{2}-1\right)\right] \\
= & a_{12} b A_{2}\left[d_{0}+r \lambda\left(c-d\left(A_{2}\right)\right)\left(2 A_{2}-1\right)\right] \\
= & a_{12} b A_{2}\left[d_{0}+\lambda\left(r c-d_{2}+b-b A_{2}\right)\left(2 A_{2}-1\right)\right] \\
= & a_{12} b A_{2}\left[-2 b \lambda A_{2}^{2}+\lambda\left(2 r c-2 d_{2}+3 b\right) A_{2}+d_{0}\right. \\
& \left.-\lambda\left(r c-d_{2}+b\right)\right]=a_{12} b A_{2} J\left(A_{2}\right) .
\end{aligned}
$$

Notice that the following facts hold: $J\left(A^{*}\right)>0$; the highest power coefficient of quadratic polynomial $J(A)$ is a negative number; $A^{-}$and $A^{+}$are two real roots of equation $J(A)=0$; and $A_{2}<A^{*}<A^{+}$. Therefore, when $A \in\left(A^{-}, A^{+}\right)$, we get $J(A)>0$, and when $A<A^{-}$or $A>A^{+}$, we get $J(A)<0$.

Thus, considering that $A_{2}<A^{*}<A^{+}$, if $A_{2}>A^{-}$; then, we have $J\left(A_{2}\right)>0$, implying $a_{3}>0$. Otherwise, if $A_{2}<A^{-}$, then $J\left(A_{2}\right)<0$, implying $a_{3}<0$.

Next, we give some conclusions on the stability of the positive equilibrium $E_{p}$.

\section{Theorem 3}

(i) Suppose that $a_{2}>0$ and $a_{1} a_{2}>a_{3}$. If $A_{2}>A^{-}$holds, then the positive equilibrium $\left(p_{2}, u_{2}, A_{2}\right)$ is locally asymptotically stable.

(ii) If $A_{2}<A^{-}$, then, the positive equilibrium $\left(p_{2}, u_{2}, A_{2}\right)$ is unstable.

Proof. For the coexistence equilibrium $E_{p}=\left(p_{2}, u_{2}, A_{2}\right)$, the characteristic equation of the linearized system (28) is as follows:

$$
\Delta(\lambda)=\left|\begin{array}{ccc}
\lambda-a_{11} & -a_{12} & d\left(A_{2}\right) \\
a_{11}+a_{31}+d_{0} & \lambda+a_{12} & -d\left(A_{2}\right)-b\left(1-A_{2}\right) \\
-a_{31} & 0 & \lambda+b A_{2}
\end{array}\right|=0 .
$$

Expanding the determinant, we get the following:

$$
\begin{aligned}
\Delta(\lambda)= & \lambda^{3}+\left(a_{12}-a_{11}+b A_{2}\right) \lambda^{2} \\
& +\left[a_{31}\left(d\left(A_{2}\right)+a_{12}\right)+a_{12} d_{0}+b A_{2}\left(a_{12}-a_{11}\right)\right] \lambda \\
& +a_{12} d_{0} b A_{2}+a_{12} a_{31} b\left(2 A_{2}-1\right) \\
= & \lambda^{3}+a_{1} \lambda^{2}+a_{2} \lambda+a_{3}=0 .
\end{aligned}
$$

(i) If $A_{2}>A^{-}$holds, then we know $a_{3}>0$ from Lemma 3. Thus we have $a_{1}>0$ from the assumptions $a_{2}>0$ and $a_{1} a_{2}>a_{3}$. Therefore, using the Routh-Hurwitz criterion, the positive equilibrium $E_{p}$ is locally asymptotically stable.

(ii) If $A_{2}<A^{-}$, then $a_{3}<0$ from Lemma 3. Hence, the characteristic equation (47) has at least one positive real root. Therefore, the positive equilibrium $\left(p_{2}, u_{2}, A_{2}\right)$ is unstable.

From (41), if $a_{12}>a_{11}$, then $a_{2}>0$, and

$$
\begin{aligned}
a_{1} a_{2}-a_{3}= & b A_{2}\left(a_{12}-a_{11}\right)^{2} \\
& +\left[b^{2} A_{2}^{2}+a_{31}\left(d+a_{12}\right)+a_{12} d_{0}\right]\left(a_{12}-a_{11}\right) \\
& +b A_{2} d a_{31}+b a_{12} a_{31}\left(1-A_{2}\right)>0 .
\end{aligned}
$$

We have the following corollary.

Corollary 1. Suppose that $A_{2}>A^{-}$holds. If $a_{12}>a_{11}$, then, the positive equilibrium $E_{p}$ is locally asymptotically stable.

\section{Effects of Weeds Removal and Other Environmental Parameters on Main Factors in Paddy Fields}

From Theorem 1, the coexistence equilibrium state $\left(p_{2}, u_{2}, A_{2}\right)$ exists when $b>d_{2}$ and (H1) hold, where $A_{2}$ is one of the solutions of equation (14), and $p_{2}$ and $u_{2}$ are computed in (19), (21), respectively.

From (14), we obtain the following:

$\frac{\mathrm{d} d_{0}}{\mathrm{~d} A}=-\lambda b \frac{(1-2 A) \ln (1-d(A) / c)+b /(r c)(A(1-A) /(1-d(A) / c))}{\ln ^{2}(1-d(A) / c)}$.

If $(1 / 2) \leq A \leq 1$, then $\left(\mathrm{d} d_{0} / \mathrm{d} A\right)<0$. Otherwise, by Lemma 2.1 in [29], we have the following:

$$
\begin{aligned}
\frac{\mathrm{d} d_{0}}{\mathrm{~d} A} & <-\lambda b \frac{(2 A-1)(d(A) /(c-d(A)))+(b / r)(A(1-A) /(c-d(A)))}{\ln ^{2}(1-d(A) / c)} \\
& =-\lambda b \frac{b A^{2}-2\left(b-d_{2}\right) A+b-d_{2}}{r(c-d(A)) \ln ^{2}(1-d(A) / c)} .
\end{aligned}
$$

Under the condition $b>d_{2}$, the discriminant of the quadratic function $b A^{2}-2\left(b-d_{2}\right) A+b-d_{2}$ is as follows:

$$
\left(-2\left(b-d_{2}\right)\right)^{2}-4 b\left(b-d_{2}\right)=-4 d_{2}\left(b-d_{2}\right)<0 \text {. }
$$

Hence, $b A^{2}-2\left(b-d_{2}\right) A+b-d_{2}>0$. It gives that $\left(\mathrm{d} d_{0} / \mathrm{d} A\right)<0$. Thus, we get the following:

$$
\frac{\mathrm{d} A}{\mathrm{~d} d_{0}}<0
$$

In the coexistence equilibrium state, $p_{2}$ can still be calculated by (13). It is not difficult to obtain that

$$
\frac{\mathrm{d} p_{2}}{\mathrm{~d} d_{0}}=\frac{b}{\lambda r(c-d(A))} \frac{\mathrm{d} A}{\mathrm{~d} d_{0}}<0 .
$$

Therefore, the biomass of herbivores and weeds decreases monotonously with the increase of the intensity of 
weeds removal. Obviously, the result that weeds biomass $p_{2}$ decreases monotonously with the increase of weeds removal intensity $d_{0}$ is consistent with the conventional understanding, but interestingly, it has nothing to do with the coupling effect coefficient $a$ of light and fertilizer, the mortality rate $d_{1}$ of weeds, and the half saturation concentration $m$ of inorganic fertilizer.

The expression (21) of the inorganic fertilizer content $u_{2}$ can also be written as follows:

$$
u_{2}=\frac{m\left(d_{0}+d_{1}+\left(d_{0} d\left(A_{2}\right) /\left(b\left(1-A_{2}\right)\right)\right)\right)}{a-\left(d_{0}+d_{1}+\left(d_{0} d\left(A_{2}\right) /\left(b\left(1-A_{2}\right)\right)\right)\right)} .
$$

Notice that the herbivores biomass $A_{2}$ is independent of parameters $a, m$, and $d_{1}$, hence $u_{2}$ is proportional to the half saturation concentration $m$ of inorganic fertilizer. If the efficiency of photosynthesis of weeds or absorbing inorganic fertilizers increases (increases $a$ ), the content of inorganic fertilizer at a steady state will decrease. If the mortality rate $d_{1}$ of weeds is increasing, the content of inorganic fertilizer could be increasing. However, the relationships between inorganic fertilizer biomass and other parameters are not so simple. We only consider the relationship between $u_{2}$ and $d_{0}$. Because $A_{2}$ is affected by the parameter $d_{0}$, we denote $d_{0}+\left(d_{0} d\left(A_{2}\right) /\left(b\left(1-A_{2}\right)\right)\right)$ by $w\left(d_{0}\right)$, that is,

$$
w\left(d_{0}\right)=d_{0}\left(1-\frac{1}{r}\right)+\frac{d_{0} d_{2}}{r b\left(1-A_{2}\right)},
$$

where $d_{0} \geq 0$. Thus, the inorganic fertilizer content $u_{2}$ can be rewritten as follows:

$$
u_{2}=\frac{m\left(d_{1}+w\left(d_{0}\right)\right)}{a-\left(d_{1}+w\left(d_{0}\right)\right)} .
$$

It is easy to obtain that

$$
\begin{aligned}
& \frac{\mathrm{d} u_{2}}{\mathrm{~d} d_{0}}=\frac{m a}{\left(a-d_{1}-w\left(d_{0}\right)\right)^{2}} \frac{\mathrm{d} w}{\mathrm{~d} d_{0}}, \\
& \frac{\mathrm{d} w}{\mathrm{~d} d_{0}}=1-\frac{1}{r}+\frac{d_{2}}{r b\left(1-A_{2}\right)}+\frac{d_{0} d_{2}}{r b\left(1-A_{2}\right)^{2}} \frac{\mathrm{d} A_{2}}{\mathrm{~d} d_{0}} .
\end{aligned}
$$

Because $A_{2}>A_{*}=1-\left(d_{2} / b\right)$, we have the following:

$$
1-\frac{1}{r}+\frac{d_{2}}{r b\left(1-A_{2}\right)}>0
$$

Therefore, $w\left(d_{0}\right)$ consists of two parts, one part increases with the increase of $d_{0}$ and the other part related to $A_{2}$ decreases with the increase of $d_{0}$. Hence, there must be a unique $d_{0}$ such that $\left(\mathrm{d} w / \mathrm{d} d_{0}\right)=0$. From this equation, we have the following:

$$
\frac{\mathrm{d} A_{2}}{\mathrm{~d} d_{0}}=-\frac{\left(1-A_{2}\right)\left[d_{2}-(1-r) b\left(1-A_{2}\right)\right]}{d_{0} d_{2}} .
$$

From (14) and (49), we get the following:

$$
\frac{\mathrm{d} d_{0}}{\mathrm{~d} A_{2}}=-\frac{d_{0}}{\lambda r\left(1-A_{2}\right)\left(c-d\left(A_{2}\right)\right.}\left[d_{0}-\lambda r\left(1-2 A_{2}\right)\left(c-d\left(A_{2}\right)\right)\right] .
$$

Multiplying (60) and (61), we obtain the following:

$$
d_{0}=\lambda r\left(c-d\left(A_{2}\right)\right)\left(1-2 A_{2}\right)+\frac{d_{2} \lambda r\left(c-d\left(A_{2}\right)\right)}{d_{2}-(1-r) b\left(1-A_{2}\right)} \text {. }
$$

By solving simultaneous equations (14) and (62), we obtain the unique $d_{0}^{*}$ that satisfies $\left(\mathrm{d} w / \mathrm{d} d_{0}\right)=0$.

Notice that

$$
\frac{\mathrm{d}^{2} w}{\mathrm{~d} d_{0}^{2}}=\frac{m a\left[d_{2}-(1-r) b\left(1-A_{2}\right)^{2}\right]}{r b\left(1-A_{2}\right)^{2}\left(a-d_{1}-w\left(d_{0}\right)\right)^{2}}>0,
$$

so $\left.\left(\mathrm{d}^{2} w / \mathrm{d} d_{0}^{2}\right)\right|_{d_{0}=d_{0}^{*}}>0$. Thus the parameter value $d_{0}^{*}$ is a minimum point of $w\left(d_{0}\right)$. Furthermore, from (57), it is easy to know that the nondifferentiable points of $u_{2}$ (such that $a-d_{1}-w\left(d_{0}\right)=0$ ) are not extreme points. Therefore, the weeds removal intensity $d_{0}^{*}$ minimizes the inorganic fertilizer content $u_{2}$. This shows that with the increase of parameter $d_{0}$, the content of inorganic fertilizer $u_{2}$ first has a downward trend, then when $d_{0}=d_{0}^{*}, u_{2}$ reaches the minimum, and then $u_{2}$ will show an upward trend.

\section{Hopf Bifurcation}

In this section, taking $m$ as the Hopf bifurcation parameter, we consider the existence of a Hopf bifurcation of system (4) at the coexistence equilibrium $E_{p}$. We always assume that the positive equilibrium $E_{p}$ exists and $A_{2}>A^{-}$holds.

From equation (14) for determining $A_{2}$ and expression (19) for calculating $p_{2}$, we know that $A_{2}$ and $p_{2}$ in equilibrium $\left(p_{2}, u_{2}, A_{2}\right)$ are independent of the half saturation concentration $m$ of inorganic fertilizer, but $u_{2}$ is related to $m$. We still find that the root $A^{-}$of equation (42) is also independent of $m$. According to (31), we can see that the parameters $a_{12}, a_{21}$, and $a_{22}$ are also related to $m$, while $a_{13}, a_{23}, a_{31}$, and $a_{33}$ are not related to $m$. From (39), we can see that the parameter $a_{11}$ is not related to $m$ either. From (41), the coefficients of the characteristic equation (47) depend on the parameter $m$. Let us set $a_{1}=a_{1}(m), a_{2}=a_{2}(m)$ and $a_{3}=a_{3}(m)$.

Lemma 3 tells us the constant term $a_{3}$ of the characteristic polynomial $\Delta(\lambda)$ is positive under the assumption $A_{2}>A^{-}$. Therefore, a Hopf bifurcation takes place when the real part of a pair of conjugate complex eigenvalues changes sign.

We define a function

$$
L(m)=a_{1}(m) a_{2}(m)-a_{3}(m)
$$

and give the following lemma [33].

Lemma 4. If there exists a critical value $m_{0}$ of a parameter $m$ such that $a_{2}\left(m_{0}\right)>0, L\left(m_{0}\right)=0$ and $L^{\prime}\left(m_{0}\right) \neq 0$, then system (4) undergoes a Hopf bifurcation at $E_{p}$ when $m$ passes $m_{0}$.

According to Lemma 4, we can know that the Hopf bifurcation critical value $m_{0}$ of the parameter $m$ is the real root of equation $L(m)=0$ which satisfies the conditions $L^{\prime}\left(m_{0}\right) \neq 0$ and $a_{2}\left(m_{0}\right)>0$. 
Because $a_{3}>0$ and $L\left(m_{0}\right)=0$, the condition $a_{2}\left(m_{0}\right)>0$ is equivalent to $a_{1}\left(m_{0}\right)=a_{12}\left(m_{0}\right)-a_{11}+b A_{2}>0$.

From (21), we get the following:

$$
\begin{aligned}
m+u_{2} & =m+\frac{m\left(b\left(1-A_{2}\right)\left(d_{0}+d_{1}\right)+d_{0} d\left(A_{2}\right)\right)}{b\left(1-A_{2}\right)\left(a-d_{0}-d_{1}\right)-d_{0} d\left(A_{2}\right)} \\
& =\frac{m a b\left(1-A_{2}\right)}{g\left(A_{2}\right)} .
\end{aligned}
$$

Substituting (65) into (33), by (31) we obtain the following:

$$
a_{12}=p_{2} \frac{a m g^{2}\left(A_{2}\right)}{\left(m a b\left(1-A_{2}\right)\right)^{2}}=\frac{A_{2} g^{2}\left(A_{2}\right)}{m a b d_{0}\left(1-A_{2}\right)} .
$$

It shows that $a_{12}$ is related to $m$. From (41), we have the following:

$$
\begin{aligned}
L(m)= & \left(a_{12}-a_{11}+b A_{2}\right)\left[a_{31}\left(d\left(A_{2}\right)+a_{12}\right)+a_{12} d_{0}+b A_{2}\left(a_{12}-a_{11}\right)\right] \\
& -a_{12} d_{0} b A_{2}-a_{12} a_{31} b\left(2 A_{2}-1\right) \\
= & \left(a_{12}-a_{11}+b A_{2}\right)\left[\left(a_{31}+d_{0}+b A_{2}\right) a_{12}+a_{31} d\left(A_{2}\right)-a_{11} b A_{2}\right] \\
& -\left[d_{0} b A_{2}+a_{31} b\left(2 A_{2}-1\right)\right] a_{12} \\
= & \left(a_{31}+d_{0}+b A_{2}\right) a_{12}^{2} \\
& +\left[b^{2} A_{2}^{2}-2 a_{11} b A_{2}+a_{31}\left(d\left(A_{2}\right)-a_{11}+b-b A_{2}\right)-a_{11} d_{0}\right] a_{12} \\
& +\left(b A_{2}-a_{11}\right)\left(a_{31} d\left(A_{2}\right)-a_{11} b A_{2}\right) .
\end{aligned}
$$

Therefore, the condition $L\left(m_{0}\right)=0$ can be rewritten as follows:

$$
\begin{aligned}
\left(a_{31}+d_{0}+b A_{2}\right) a_{12}^{2}+ & {\left[b^{2} A_{2}^{2}-2 a_{11} b A_{2}+a_{31}\left(d\left(A_{2}\right)-a_{11}\right.\right.} \\
& \left.\left.+b-b A_{2}\right)-a_{11} d_{0}\right] \\
& -a_{12}+\left(b A_{2}-a_{11}\right)\left(a_{31} d\left(A_{2}\right)\right. \\
& \left.-a_{11} b A_{2}\right)=0 .
\end{aligned}
$$

Of all the parameters of the equation (68), only $a_{12}$ is related to the bifurcation parameter $m$. Therefore, by calculating $a_{12}$ from equation (68), the bifurcation parameter $m$ can be determined by (66).

In addition, from (31), (39), and (66), we obtain that

$$
\begin{aligned}
L^{\prime}(m) & =\left(2\left(a_{31}+d_{0}+b A_{2}\right) a_{12}+b^{2} A_{2}^{2}-2 a_{11} b A_{2}+a_{31}\left(d\left(A_{2}\right)-a_{11}+b-b A_{2}\right)-a_{11} d_{0}\right) a_{12}^{\prime}(m) \\
& =-\left(2\left(a_{31}+d_{0}+b A_{2}\right) a_{12}+b^{2} A_{2}^{2}-2 a_{11} b A_{2}+a_{31}\left(d\left(A_{2}\right)-a_{11}+b-b A_{2}\right)-a_{11} d_{0}\right) \times \frac{A_{2} g^{2}\left(A_{2}\right)}{m^{2} a b d_{0}\left(1-A_{2}\right)} .
\end{aligned}
$$

Notice that $g\left(A_{2}\right)>0$, the condition $L^{\prime}\left(m_{0}\right) \neq 0$ can be replaced by the following condition:

$$
a_{12} \neq-\frac{b^{2} A_{2}^{2}-2 a_{11} b A_{2}+a_{31}\left(d\left(A_{2}\right)-a_{11}+b-b A_{2}\right)-a_{11} d_{0}}{2\left(a_{31}+d_{0}+b A_{2}\right)} .
$$

According to Lemma 4, we can get the existence of a Hopf bifurcation of system (4).

Theorem 4. Suppose that conditions (H1), $b>d_{2}$ and $A_{2}>A^{-}$hold. If equation (68) has a positive real root $a_{12}$ satisfying (70) and $a_{12}>a_{11}-b A_{2}$, then system (4) undergoes a Hopf bifurcation at $E_{p}$ when $m$ passes $m_{0}$, where

$$
m_{0}=\frac{A_{2} g^{2}\left(A_{2}\right)}{a b a_{12} d_{0}\left(1-A_{2}\right)} .
$$

Hence, once $a_{12}$ is obtained from equation (68), we can calculate the critical value $m_{0}$ with (71), which is obtained by (66).

According to the conditions of Theorem 4, we summarize the process of calculating the critical value $m_{0}$ of the Hopf bifurcation as follows:

(1) If the condition $b>d_{2}$ holds, then $A_{2}$ is obtained by solving equation (14) and $p_{2}$ is calculated by (19). If the condition (H1) is validated, then system (4) has a positive equilibrium $E_{p}$.

(2) Find the root $A^{-}$of the quadratic equation (42) and verify the assumption $A_{2}>A^{-}$.

(3) Calculate $a_{11}$ with (39) and $a_{31}$ with (31).

(4) Solve the quadratic equation (68). If there is a positive real root, then we obtain the parameter $a_{12}$. 
(5) Verify the conditions (70) and $a_{12}>a_{11}-b A_{2}$. If one of them is not valid, the critical value $m_{0}$ can not be calculated.

(6) Calculate the critical value $m_{0}$ with (71).

Remark 1. If the quadratic equation (68) has two positive real roots for $a_{12}$, and both of them satisfy all the conditions of Theorem 4, then there are two Hopf bifurcations at the positive equilibrium point $E_{p}$.

\section{An Example}

In system (4), take a set of simulation parameters $a=40$, $c=6, r=0.5, \lambda=6, d_{0}=0.3, d_{1}=0.2$, and $d_{2}=0.7$. We consider two cases of parameter $b=1$ and $b=4.4$, which satisfy the condition $b>d_{2}$.

By the process of calculating the critical value $m_{0}$ of a Hopf bifurcation in Section 6, we obtain the calculation results of the Hopf bifurcation, which are listed in Table 1. From the line of condi in the table and $a=40$, it follows that hypothesis (H1) holds, so there is a positive equilibrium point in system (4) by Theorem 1. It is easy to see from the second and seventh rows of Table 1 that $A_{2}>A^{-}$. From the second line to the fourth line counted backward in the table, we obtain $a_{12}>a_{11}-b A_{2}$ and $a_{12} \neq$ cond $i_{2}$. Therefore, the conditions in Theorem 4 are all satisfied.

From Table 1, we see that the critical value $m_{0} \approx 0.020224$ when parameter $b=1$. Therefore, system (4) undergoes a Hopf bifurcation at $E_{p}$ when $m$ passes $m_{0} \approx 0.020224$. If we let $m=0.02<m_{0} \approx 0.020224$, system (4) has a positive equilibrium $E_{p} \approx(0.0433,0.0774,0.9868)$. From (41), we get $a_{1} \approx 0.7533, a_{2} \approx 69.7690>0$, and $a_{3} \approx 49.8515$. Hence, $a_{1} a_{2}-a_{3} \approx 2.7039>0$. According to Theorem 3 , the positive equilibrium $E_{p} \approx(0.0433,0.0774,0.9868)$ is locally asymptotically stable (see Figure 1). Therefore, if $m$ passes $m_{0} \approx 0.020224$ from 0.02 , then the positive equilibrium $E_{p}$ loses its stability, and system (4) has a periodic solution (see Figure 2, where $m=0.021)$.

When the parameter $b=4.4$, from Table 1 , we still know that system (4) has two critical values of the Hopf bifurcation $m_{0} \approx 0.024358$ or $m_{0} \approx 4.318921$. Therefore, system (4) undergos a Hopf bifurcation at $E_{p}$ when $m$ passes $m_{0} \approx 0.024358$. It also undergoes the other Hopf bifurcation when $m$ passes $m_{0} \approx 4.318921$. If we let $m=0.02<m_{0} \approx 0.024358$, system (4) has a positive equilibrium $E_{p} \approx(0.0433,0.0813,0.9970)$. From (41), we get $a_{1} \approx 3.8315, a_{2} \approx 64.3037>0$, and $a_{3} \approx 208.7775$. Thus, we have $a_{1} a_{2}-a_{3} \approx 37.6027>0$. Therefore, according to Theorem 3 , the positive equilibrium $E_{p} \approx(0.0433,0.0813,0.9970)$ is locally asymptotically stable, which means there exists a periodic solution of system (4) when $m>m_{0} \approx 0.024358$. If we let $m=4.32>m_{0} \approx 4.318921$, system (4) has a positive equilibrium $E_{p} \approx(0.0433,17.5696,0.9970)$. From (41), we get $a_{1} \approx 0.4710, a_{2} \approx 2.0527>0$, and $a_{3} \approx 0.9666$. Then, we have $a_{1} a_{2}-a_{3} \approx 1.9931 \times 10^{-4}>0$. Therefore, according to Theorem 3, the positive equilibrium
Table 1: The calculation results of the Hopf bifurcation.

\begin{tabular}{lcc}
\hline Items & $b=1$ & $b=4.4$ \\
\hline$A_{2}$ & 0.9868 & 0.9970 \\
$p_{2}$ & 0.0433 & 0.0433 \\
cond $i_{1}{ }^{(1)}$ & 31.7831 & 32.1058 \\
$A^{-}$ & 0.4911 & 0.4945 \\
$a_{11}$ & 3.8907 & 3.9316 \\
$a_{31}(2)$ & 13.6962 & 13.8371 \\
$a_{12}$ & 3.6166 & $0.0156,2.7721$ \\
$a_{11}-b A_{2}$ & 2.9038 & -0.4553 \\
cond $i_{2}{ }^{(3)}$ & 1.4071 & 1.3939 \\
$m_{0}$ & 0.020224 & $4.318921,0.024358$ \\
\hline
\end{tabular}

${ }^{(1)}$ cond $i_{1}=d_{0}+d_{1}+\left(d_{0} d\left(A_{2}\right) /\left(b\left(1-A_{2}\right)\right)\right) .{ }^{(2)} a_{12}$ is the positive real roots of equation (68). ${ }^{(3)}$ cond $i_{2}$ is the right side of (70).

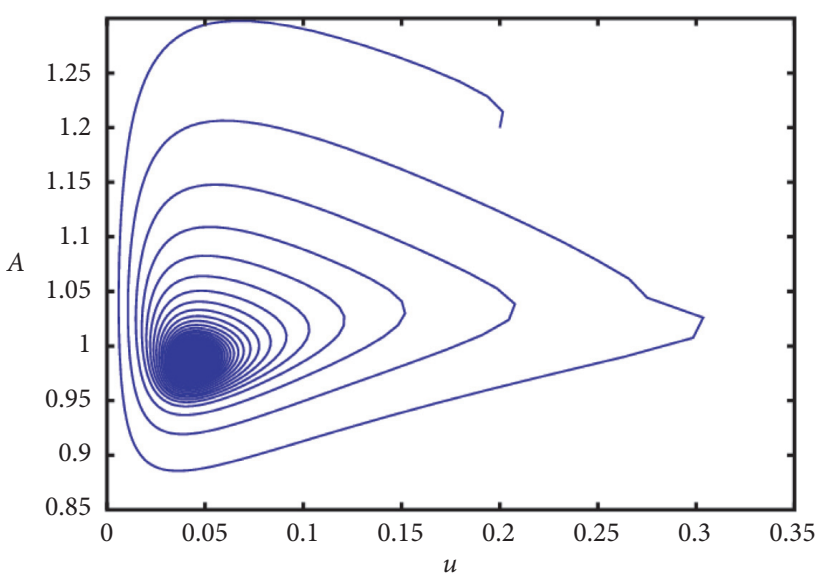

Figure 1: The phase diagram of $u-A$ of system (4) with $a=40$, $c=6, \lambda=6, \quad r=0.5, \quad b=1, \quad d_{0}=0.3, d_{1}=0.2, d_{2}=0.7$ and $m=0.02$. It describes the asymptotic stability of the equilibrium $(0.0433,0.0774,0.9868)$.

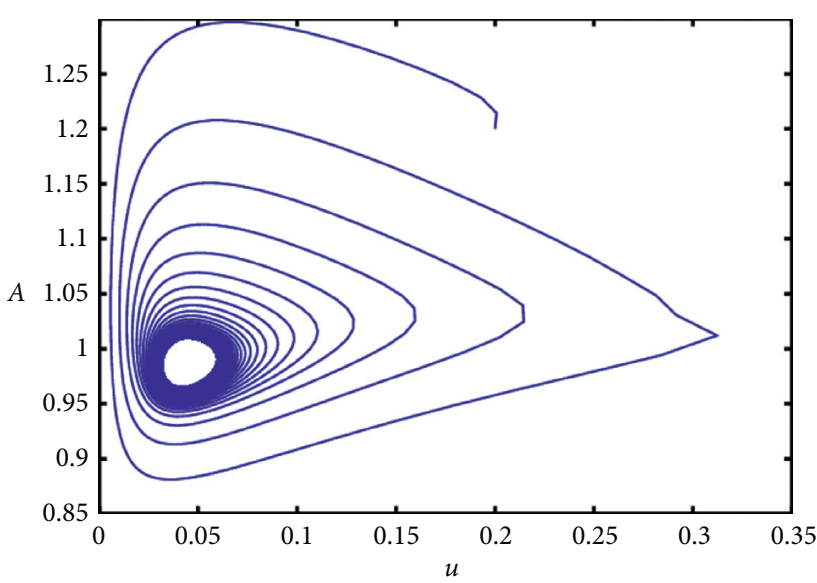

FIgURe 2: The phase diagram of $u-A$ of system (4) with $a=40$, $c=6, \lambda=6, \quad r=0.5, \quad b=1, d_{0}=0.3, d_{1}=0.2, d_{2}=0.7$, and $m=0.021$. It describes the instability of the positive equilibrium and indicates a positive periodic solution exists in the system (4).

$E_{p} \approx(0.0433,17.5696,0.9970)$ is locally asymptotically stable, implying that there exists a periodic solution of system (4) when $m<m_{0} \approx 4.318921$. 


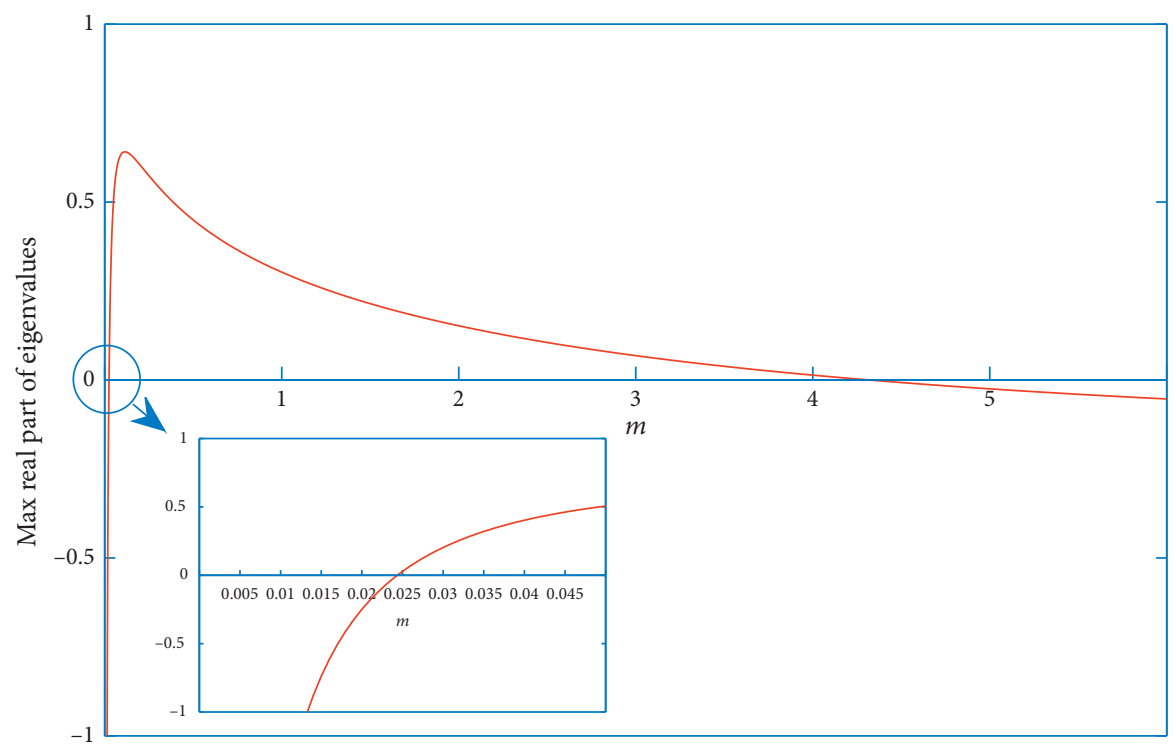

FIGURE 3: The relationship curve between the maximum real part of the eigenvalue of the characteristic equation (47) and parameter $m$ with $a=40, c=6, \lambda=6, r=0.5, b=4.4, d_{0}=0.3, d_{1}=0.2$ and $d_{2}=0.7$.

Combining the above calculation results when $b=4.4$, we see that when the parameter $m$ passes through the critical values 0.024358 and 4.318921 from left to right, the positive equilibrium of system (4) undergoes a process of change from stability to instability and then to stability. Figure 3 depicts the relationship between the maximum real part of the eigenvalue of the characteristic equation (47) with $b=$ 4.4 and the parameter $m$. It can be seen from Figure 3 that the maximum real part of the eigenvalue is positive in the interval $[0.024358,4.318921]$, indicating that system (4) is unstable in this range, while the maximum real part of the system outside the interval is negative, which indicates that system (4) is stable in this range. This is consistent with our calculation in the above.

\section{Conclusion}

By establishing a differential equation model of paddy ecosystem in the fallow season with weeds removal and analyzing its stability and Hopf bifurcation, we found the interaction among weeds, inorganic fertilizer, and herbivores in this system.

In a paddy ecosystem in the fallow season, because of the human management activities of weeds removal and animal farming, the extinction of weeds and herbivores may occur. However, the extinction state of weeds and herbivores is unstable. From (30), we know that as long as the growth rate of herbivores is higher than their excretion rate, the extinction of herbivores in paddy fields can be avoided.

Furthermore, when $b>d_{2}$, if the coupling effect coefficient of light and fertilizer $a$ is higher than the sum of weeds removal intensity $d_{0}$, weeds mortality $d_{1}$, and $\left(d_{0} d\left(A_{2}\right) /\left(b\left(1-A_{2}\right)\right)\right)$ (assumption $\left.(\mathrm{H} 1)\right)$, the coexistence equilibrium $E_{p}$ can be found in the system.

Our results are obtained under the condition of $d_{0}>0$. When weeds are not removed, i.e., $d_{0}=0$, the situation is relatively simple. Xiang et al. has considered the case of $d_{0}=$ 0 in [29]. They obtained that $p_{2}$ and $u_{2}$ as follows:

$$
\begin{aligned}
& p_{2}=-\frac{1}{\lambda} \ln \left(1-\frac{d_{2}}{r c}\right), \\
& u_{2}=\frac{m\left(r p_{2} d_{1}+d_{2}\right)}{r p_{2}\left(a-d_{1}\right)-d_{2}} .
\end{aligned}
$$

Obviously, they need conditions $r c>d_{2}$ and $p_{2}>d_{2} /\left(r\left(a-d_{1}\right)\right)$. In Theorem 1 , we replaced their condition $p_{2}>d_{2} /\left(r\left(a-d_{1}\right)\right)$ with (H1) and deleted condition $r c>d_{2}$. Thus, our results include the case of $r c \leq d_{2}$. Because conditions (H1) and (25) are equivalent, from (25), we know that the intensity of weeds removal $d_{0}$ should not be too high $\left(d_{0}<a-d_{1}\right)$.

By using the Routh-Hurwitz criterion, we obtain the conditions for local asymptotic stability of the positive equilibrium $E_{p}$, in which the condition $A_{2}>A^{-}$is used to guarantee the coefficient $a_{3}>0$. If $d_{0}=0$, then we have $A_{2}=$ 1 from (6), so the coefficient $a_{3}=a_{12} a_{31} b>0$ is natural. So there is no requirement for the lower bound of $A_{2}$ in Theorem 2.5 for the local stability of positive equilibrium in [29]. However, if weeds removal is considered in the system, then whether $a_{3}$ is positive or negative is affected by the value $A_{2}$. From (41), we see that $A_{2}$ should not be too small, otherwise $a_{3}$ will be negative. This is why we propose the condition $\mathrm{A}_{2}>\mathrm{A}^{-}$in Theorem 3 .

When the coefficients $a_{i}$ of cubic characteristic polynomial (47) are positive, the Hopf bifurcation may be generated when $a_{1} a_{2}=a_{3}$. According to this principle, we obtain the Hopf bifurcation conditions of system (4) at the positive equilibrium $E_{p}$, and give the Hopf bifurcation critical value formula (71) with $m$ being the bifurcation parameter. The half saturation concentration of inorganic fertilizer is a key parameter in Michaelis-Menten uptake 
kinetics, which is affected by species of weeds. A Hopf bifurcation appears at a positive equilibrium $E_{p}$ when the parameter $m$ passes through the critical value $m_{0}$, which indicates that different kinds of weeds in paddy field may lead to different dynamic properties of the paddy ecosystem. It is necessary to know the half saturation concentration of inorganic fertilizer for each weeds, so as to grasp the stability of the paddy ecosystem in advance. From our numerical simulation results, the paddy ecosystem with high half saturation concentration of weeds is more prone to Hopf bifurcation.

From the analysis in Section 5, we also see that weeds removal can reduce the biomass of weeds in an equilibrium state. However, this activity also reduces the biomass of herbivores in paddy fields. If the intensity of weeds removal is $d_{0}^{*}$, the soil inorganic fertilizer content is the lowest. The weeds removal intensity is too small to achieve the purpose of weeding. Therefore, the intensity of weeds removal should be greater than the minimum point $d_{0}^{*}$ as far as possible. But considering that $d_{0}$ is restricted by condition ( $\left.\mathrm{H} 1\right)$, it has an upper bound. For example, we can know from (25) that $d_{0}$ cannot exceed $a-d_{1}$ at least. If the minimum point $d_{0}^{*}$ is greater than the upper bound, then we should try to use lowintensity weeding. If $d_{0}^{*}$ is less than the upper bound, the intensity of weeds removal should be close to the upper bound and not exceed it.

\section{Data Availability}

No data were used to support this study. In our research, we use numerical simulation to verify our main results. The system parameters used in the simulation have been included within this paper.

\section{Conflicts of Interest}

All authors declare no conflicts of interest in this paper.

\section{Acknowledgments}

This work was partly supported by Hunan Province Science and Technology Projects (grants 2015JC3101) and the Hunan Provincial Natural Science Foundation (No. 2019JJ50222).

\section{References}

[1] E. A. Martin, B. Seo, C.-R. Park, B. Reineking, and I. SteffanDewenter, "Scale-dependent effects of landscape composition and configuration on natural enemy diversity, crop herbivory, and yields," Ecological Applications, vol. 26, no. 2, pp. $448-462,2016$.

[2] A. G. Firth, B. H. Baker, J. P. Brooks, R. Smith, R. B. Iglay, and J. Brian Davis, "Low external input sustainable agriculture: winter flooding in rice fields increases bird use, fecal matter and soil health, reducing fertilizer requirements," Agriculture, Ecosystems \& Environment, vol. 300, Article ID 106962, 2020.

[3] M. Dainese, E. Martin, M. Aizen et al., "A global synthesis reveals biodiversity-mediated benefits for crop production," Science Advances, vol. 5, no. 10, Article ID eaaz0121, 2019.
[4] T. Wen, B. Zhao, and J. Zhang, "Emission pathways and influencing factors for $\mathrm{CH}_{4}$ and $\mathrm{N}_{2} \mathrm{O}$ from rice-duck farming," Journal of Agro-Environment Science, vol. 39, no. 7, pp. 1442-1450, 2020.

[5] Q. Teng, X. Hu, F. Luo, J. Wang, and D. Zhang, "Promotion of rice-duck integrated farming in the water source areas of shanghai: its positive effects on reducing agricultural diffuse pollution," Environmental Earth Sciences, vol. 78, no. 5, Article ID 171, 2019.

[6] Y. Liao, Y. Huang, N. Han, C. Zou, D. Shi, and D. Jiang, "Analysis of population diversity of fungi and bacteria in rice rhizosphere soil under rice-duck farming model," Journal of Agriculture, vol. 50, no. 1, pp. 59-67, 2019.

[7] H. Gao, Z. Sha, F. Wang et al., "Nitrogen leakage in a riceduck co-culture system with different fertilizer treatments in China," Science of The Total Environment, vol. 686, no. 10, pp. 555-567, 2019.

[8] S. Abbas, M. Sen, and M. Banerjee, "Almost periodic solution of a non-autonomous model of phytoplankton allelopathy," Nonlinear Dynamics, vol. 67, no. 1, pp. 203-214, 2012.

[9] D. Jia, T. Zhang, and S. Yuan, "Pattern dynamics of a diffusive toxin producing phytoplankton-zooplankton model with three-dimensional patch," International Journal of Bifurcation and Chaos, vol. 29, no. 4, Article ID 1930011, 2019.

[10] X.-Y. Meng and Y.-Q. Wu, "Dynamical analysis of a fuzzy phytoplankton-zooplankton model with refuge, fishery protection and harvesting," Journal of Applied Mathematics and Computing, vol. 63, no. 1-2, pp. 361-389, 2020.

[11] C. Dai, M. Zhao, and H. Yu, "Dynamics induced by delay in a nutrient-phytoplankton model with diffusion," Ecological Complexity, vol. 26, pp. 29-36, 2016.

[12] K. P. Das, P. Roy, P. Karmakar, and S. Sarkar, "Role of viral infection in controlling planktonic blooms-conclusion drawn from a mathematical model of phytoplankton-zooplankton system," Differential Equations and Dynamical Systems, vol. 28, no. 2, pp. 381-400, 2020.

[13] E. E. Hofmann and J. W. Ambler, "Plankton dynamics on the outer southeastern U.S. continental shelf. Part II: a timedependent biological model," Journal of Marine Research, vol. 46, no. 4, pp. 883-917, 1988.

[14] S. R.-J. Jang and E. J. Allen, "Deterministic and stochastic nutrient-phytoplankton-zooplankton models with periodic toxin producing phytoplankton," Applied Mathematics and Computation, vol. 271, pp. 52-67, 2015.

[15] Y. Sekerci and S. Petrovskii, "Mathematical modelling of plankton-oxygen dynamics under the climate change," Bulletin of Mathematical Biology, vol. 77, no. 12, pp. 2325-2353, 2015.

[16] A. Sharma, A. K. Sharma, and K. Agnihotri, "Analysis of a toxin producing phytoplankton-zooplankton interaction with Holling IV type scheme and time delay," Nonlinear Dynamics, vol. 81, no. 1-2, pp. 13-25, 2015.

[17] A. K. Sharma, A. Sharma, and K. Agnihotri, "Bifurcation behaviors analysis of a plankton model with multiple delays," International Journal of Biomathematics, vol. 9, no. 6, pp. 113-137, 2016.

[18] Z. Jiang, X. Bi, T. Zhang, and B. G. Sampath Aruna Pradeep, "Global hopf bifurcation of a delayed phytoplankton-zooplankton system considering toxin producing effect and delay dependent coefficient," Mathematical Biosciences and Engineering, vol. 16, no. 5, pp. 3807-3829, 2019.

[19] H. Zhang and B. Niu, "Dynamics in a plankton model with toxic substances and phytoplankton harvesting," 
International Journal of Bifurcation and Chaos, vol. 30, no. 2, Article ID 2050035, 2020.

[20] F. Li, S. Zhang, and X. Meng, "Dynamics analysis and numerical simulations of a delayed stochastic epidemic model subject to a general response function," Computational and Applied Mathematics, vol. 38, no. 2, Article ID 95, 2019.

[21] G. Liu, H. Qi, Z. Chang, and X. Meng, "Asymptotic stability of a stochastic may mutualism system," Computers \& Mathematics with Applications, vol. 79, no. 3, pp. 735-745, 2020.

[22] A. Maiti, B. Patra, and G. P. Samanta, "Sterile insect release method as a control measure of insect pests: a mathematical model," Journal of Applied Mathematics and Computing, vol. 22, no. 3, pp. 71-86, 2006.

[23] A. Maiti, A. K. Pal, and G. P. Samanta, "Usefulness of biocontrol of pests in tea: a mathematical model," Mathematical Modelling of Natural Phenomena, vol. 3, no. 4, pp. 96-113, 2008.

[24] A. Mondal, A. K. Pal, and G. P. Samanta, "Analysis of a delayed eco-epidemiological pest-plant model with infected pest," Biophysical Reviews and Letters, vol. 14, no. 2, pp. 141-170, 2019.

[25] A. Mondal, A. K. Pal, and G. P. Samanta, "Rich dynamics of non-toxic phytoplankton, toxic phytoplankton and zooplankton system with multiple gestation delays," International Journal of Dynamics and Control, vol. 8, no. 1, pp. 112-131, 2020.

[26] D. Sahoo, S. Mondal, and G. P. Samanta, "Interaction among toxic phytoplankton with viral infection and zooplankton in presence of multiple time delays," International Journal of Dynamics and Control, pp. 1-26, 2020.

[27] H. Zhao, X. Huang, and X. Zhang, "Hopf bifurcation and harvesting control of a bioeconomic plankton model with delay and diffusion terms," Physica A: Statistical Mechanics and its Applications, vol. 421, pp. 300-315, 2015.

[28] M. Xiang, Z. Wu, and T. Zhou, "Stability of a paddy ecosystem in fallow season," Journal of Biomathematics, vol. 32, no. 1, pp. 49-56, 2017, in Chinese.

[29] M. Xiang, Z. Wu, and T. Zhou, "Analysis of the interaction among weed, inorganic fertilizer and herbivore in paddy ecosystem in fallow season," International Journal of Biomathematics, vol. 10, no. 8, pp. 249-259, 2017.

[30] X. Zhou, Z. Wu, Z. Wang, and T. Zhou, "Stability and Hopf bifurcation analysis in a fractional order delayed paddy ecosystem," Advances in Difference Equations, vol. 2018, no. 1, Article ID 315, 2018.

[31] Z. Wu, Y. Wang, X. Zhou, and T. Zhou, "Analysis of the interaction among rice, weeds, inorganic fertilizer, and a herbivore in a composite farming paddy ecosystem," Mathematical Biosciences, vol. 300, pp. 145-156, 2018.

[32] K. Zheng, X. Zhou, Z. Wu, Z. Wang, and T. Zhou, "Hopf bifurcation controlling for a fractional order delayed paddy ecosystem in the fallow season," Advances in Difference Equations, vol. 2019, no. 1, Article ID 307, 2019.

[33] W. Liu, "Criterion of Hopf bifurcations without using eigenvalues," Journal of Mathematical Analysis and Applications, vol. 182, no. 1, pp. 250-256, 1994. 